\title{
Feedback stabilization of control systems described by a class of nonlinear differential-algebraic equations
}

\author{
N. Harris McClamroch * \\ Department of Aerospace Engineering, The University of Michi- \\ gan, Ann Arbor, MI 48109, U.S.A.
}

Received 21 October 1989

Revised 20 January and 26 March 1990

\begin{abstract}
A theoretical framework for the stabilization of control systems defined by a class of nonlinear differential-algebraic equations is established. Assumptions are stated which guarantee that the nonlinear differential-algebraic equations can be described by a nonlinear control system defined on a smooth manifold. A procedure for obtaining a local state realization is developed. Conditions for local stabilization of a single equilibrium solution, including one set of conditions which can easily be checked using standard computations, are indicated.
\end{abstract}

Keywords: Differential-algebraic equations; stabilization; state realization.

\section{Introduction}

An important research area is concerned with control of nonlinear systems described by ordinary differential equations with certain outputs required to be identically zero. Such research has exposed the concepts of nonlinear zeros and has had important ramifications in smooth stabilization, feedback linearization and disturbance decoupling $[4,6,9]$. This research has led to the introduction of the concept of zero dynamics; recently, the more general concept of clamped dynamics for a nonlinear control system has been introduced in [15]. A related area of research has been concerned with initial value problems defined by nonlinear differential-algebraic equations; existence

* This research was supported in part by NSF Grant MSM8722266. and uniqueness results have been developed in [14] and numerical integration issues have been considered in [3]. During the same period, there have been developments in the control of mechanical systems with holonomic constraints $[11,12]$. A recent paper [1] has examined several control theoretic issues which pertain to both holonomic and nonholonomic constraints in a very general form. Stabilization of mechanical systems with classical nonholonomic constraints has been considered in [2].

In this paper, we study generalizations of the previous models for constrained mechanical systems $[1,2,11,12]$ using the formalisms developed for studying zero dynamics, clamped dynamics and differential-algebraic systems. Our formulation is a special case of the clamped dynamics formalism introduced in [15], but our specific assumptions allow us to give a precise characterization of the nonlinear systems studied. The special structure of the systems considered in this paper, with the associated assumptions, is sufficiently general to be applicable to a large and important class of electromechanical systems where constraints arise naturally from physical considerations [10].

2. Control systems described by a class of nonlinear differential-algebraic equations

We begin by introducing a smooth nonlinear system defined by

$$
\begin{aligned}
& \dot{x}=f(x)+\sum_{i=1}^{m} g_{i}(x) v_{i}+\sum_{i=1}^{p} h_{i}(x) u_{i}, \\
& y_{i}=k_{i}(x), \quad i=1, \ldots, m, \\
& z_{i}=l_{i}(x), \quad i=1, \ldots, p,
\end{aligned}
$$


where $x \in M$, with $M$ an open subset of $R^{n}$,

$v=\left(v_{1}, \ldots, v_{m}\right) \in R^{m}, \quad u=\left(u_{1}, \ldots, u_{p}\right) \in R^{p}$,

$y=\left(y_{1}, \ldots, y_{m}\right) \in R^{m}, \quad z=\left(z_{1}, \ldots, z_{p}\right) \in R^{p}$.

Here $f(x), g_{i}(x), i=1, \ldots, m, h_{i}(x), i=1, \ldots, p$, are smooth vector fields on $M$ and $k_{i}(x), i=$ $1, \ldots, m$ and $l_{i}(x), i=1, \ldots, p$, are smooth functions on $M$. In this formulation there are $m+p$ input variables $(v, u)$ and $m+p$ output variables $(y, z)$. The input functions

$v:[0, \infty) \rightarrow R^{m}$ and $u:[0, \infty) \rightarrow R^{p}$

are integrable on any finite interval.

We are especially interested in the constrained dynamics, i.e. the dynamics of equations (2.1)(2.3) that are consistent with the imposition of the constraints: $y_{i}=0, i=1, \ldots, m$. We subsequently refer to $v$ as the constraint input variables, to $u$ as the control input variables, to $y$ as the constraint output variables, and to $z$ as the control output variables. We now define in a more formal way these constrained dynamics. Our definitions follow the existing literature on nonlinear control systems.

A smooth manifold $N \subset M$ is an output nulling controlled invariant manifold for equations (2.1)(2.2) if there exists a smooth feedback such that $N$ is an invariant manifold for the closed loop equations for each input function $u:[0, \infty) \rightarrow R^{p}$ and the output functions $k_{i}(x), i=1, \ldots, m$, are zero on $N$. If there is an output nulling controlled invariant manifold $N$ for equations (2.1)-(2.2) we refer to the implicitly defined system on $N$, with input $\mathrm{u}$ and output $z$, as a constrained system. This system is formally described by the nonlinear differential-algebraic equations

$$
\begin{aligned}
& \dot{x}=f(x)+\sum_{i=1}^{m} g_{i}(x) v_{i}+\sum_{i=1}^{p} h_{i}(x) u_{i}, \\
& k_{i}(x)=0, \quad i=1, \ldots, m, \\
& z=l_{i}(x), \quad i=1, \ldots, p .
\end{aligned}
$$

The constrained system can also be viewed as the system implicitly defined by the differentialalgebraic equations (2.4)-(2.6); such an implicit definition is consistent with the perspective for singular or implicit differential equations, of which equations (2.4)-(2.6) represent an important special class. We emphasize that these equations do not provide a state realization for the constrained system, and a suitable output nulling controlled invariant manifold is not explicitly identified. Consequently, the existence of 'well defined' solutions of equations (2.4)-(2.6) requires justification.

Our fundamental assumptions for the class of nonlinear constrained systems defined by equations (2.4)-(2.6) are now introduced. Assume that $x_{0} \in M$ and there are finite positive integers $r_{i}$, $i=1, \ldots, m$, such that:

(A1) $L_{g_{j}} L_{f}^{k} k_{i}(x)=0$, for all $k=0, \ldots, r_{i}-2, i=$ $1, \ldots, m, j=1, \ldots, m$, and $x \in M \cap N\left(x_{0}\right)$.

(A2) rank $A\left(x_{0}\right)=m$ where $A(x)$ is an $m \times m$ matrix whose element on the $i$-th row and the $j$-th column is

$a_{i j}(x)=L_{g_{j}} L_{f}^{r_{i}-1} k_{i}(x)$.

(A3) $L_{h_{j}} L_{f}^{k} k_{i}(x)=0$, for all $k=0, \ldots, r_{i}-2, i=$ $1, \ldots, m, j=1, \ldots, p$, and $x \in M \cap N\left(x_{0}\right)$.

As usual $N\left(x_{0}\right)$ denotes a neighborhood of $x_{0}$ and the notation $L_{f} k_{i}(x)$ indicates the directional derivative of $k_{i}(x)$ along the vector field $f(x)$ and $L_{f}^{k} k_{i}(x)=L_{f}\left(L_{f}^{k-1} k_{i}(x)\right)$.

Assumptions (A1)-(A3) are essentially that the constraint relative degrees of $(f, g, k)$ are well defined and finite, that the decoupling matrix $A\left(x_{0}\right)$ has full row rank and that the control relative degrees of $(f, h, k)$ are well defined and finite and no less than the constraint relative degrees. In other words, the constraint relative degree vector of $(f, g, k)$ is $\left(r_{1}, \ldots, r_{m}\right)$ at $x_{0} \in M$ and is not greater than the control relative degree vector of $(f, h, k)$, componentwise, where

$g(x)=\left[g_{1}(x), \ldots, g_{m}(x)\right]$,

$h(x)=\left[h_{1}(x), \ldots, h_{p}(x)\right]$

[6, p.235]. If $r_{i}=r, i=1, \ldots, m$, these assumptions are equivalent to the assumption that the index, as defined for nonlinear singular (or implicit) equations as in [3], is $r+1$ at $x_{0} \in M$. We mention that models of mechanical systems with holonomic constraints, as studied in [11,12], are examples of constrained systems with global constraint 
relative degree vector $(2, \ldots, 2)$, and that the models of mechanical systems with classical nonholonomic constraints, as studied in [2], are examples of constrained systems with global constraint relative degree vector $(1, \ldots, 1)$.

We now assert that the differential-algebraic equations (2.4)-(2.5) are well posed in the sense that an associated initial value problem has a unique solution, at least locally. Suppose that $u(t)$ is a given integrable function. A pair of vector functions $(x(t), v(t))$ defined on an interval $[0, T)$ is a solution of the initial value problem defined by equations (2.4) $-(2.5)$ and the initial condition data $x_{1}$ if $x(t)$ is differentiable, $v(t)$ is integrable, the vector function $(x(t), v(t))$ satisfy the differential-algebraic equations (2.4)-(2.5) almost everywhere on their domain of definition and $x(0)$ $=x_{1}$.

Theorem 2.1. Define

$$
\begin{aligned}
N^{*}=\{ & x \in M \cap N\left(x_{0}\right) \mid \\
& L_{f}^{k} k_{i}(x)=0, k=0,1, \ldots, r_{i}-1, \\
& i=1, \ldots, m\} .
\end{aligned}
$$

Then $N^{*}$ is an $n-\left(r_{1}+\cdots+r_{m}\right)$ dimensional integral manifold of the maximal controlled invariant distribution contained in

$\operatorname{ker}\left\{\mathrm{d} k_{1}(x), \ldots, \mathrm{d} k_{m}(x)\right\}$.

Assume that $x_{0} \in N^{*}$ and the input function $u:[0, \infty) \rightarrow R^{p}$ is a given integrable function. There is a neighborhood $N\left(x_{0}\right)$ of $x_{0}$ such that if the initial condition data satisfy $x_{1} \in N^{*}$, then there exists a unique solution (at least locally defined) of the initial value problem defined by the differential algebraic equations (2.4)-(2.5) and the solution satisfies $x(t) \in N^{*}$ for each $t$ for which the solution is defined.

Proof. The properties of $N^{*}$ follow from the stated assumptions using the development in [6, pp. 236-237]. Following the development in [14], it is easily demonstrated that for each $u:[0, \infty) \rightarrow$ $R^{p}$ the differential-algebraic equations (2.4)-(2.5) define a smooth vector field on the manifold $N^{*}$; the results follow according to [5]. A more detailed and direct proof of this result is given in [10].

We thus make the additional assumption that $x_{0} \in N^{*}$ in our subsequent development. Since the differential-algebraic equations (2.4)-(2.5) define, for each integrable input function $u:[0, \infty)$ $\rightarrow R^{p}$, a smooth vector field on the manifold $N^{*}$, a number of others results could be stated, including conditions for continuous dependence of the solution on initial conditions and parameters, conditions for non-existence of finite escape times, etc. Such results are important, but they are not given here since they are easily obtained. The stated assumptions guarantee that the space of solutions of the differential-algebraic equations (2.4)-(2.5) lie in the manifold $N^{*}$ defined by (2.7). An algorithmic specification of consistent initial data for a more general class of differential-algebraic equations has recently been given in [13].

3. A feedback stabilization problem for systems described by nonlinear differential-algebraic equations

Our particular interest is to study feedback systems which are defined using equations (2.4)(2.6) and a feedback map defined by $u: N^{*} \times R^{p}$ $\rightarrow R^{p}$ where

$u_{i}=\gamma_{i}(x)+\sum_{j=1}^{p} \sigma_{i j}(x) w_{j}, \quad i=1, \ldots, p$

here $w=\left(w_{1}, \ldots, w_{p}\right) \in R^{p}$ is the closed loop input. The closed loop constrained system is defined by the nonlinear differential-algebraic equations

$$
\begin{aligned}
& \dot{x}=\left\{f(x)+\sum_{i=1}^{p} h_{i}(x) \gamma_{i}(x)\right\} \\
& \quad+\sum_{i=1}^{m} g_{i}(x) v_{i}+\sum_{i=1}^{p} \sum_{j=1}^{p} \sigma_{j i}(x) h_{j}(x) w_{i}, \\
& k_{i}(x)=0, \quad i=1, \ldots, m, \\
& z_{j}=l_{i}(x), \quad i=1, \ldots, p .
\end{aligned}
$$

It is easy to show that Assumptions (A1)-(A3) also are satisfied at $x_{0} \in N^{*}$ for all closed loop equations of the form (3.2)-(3.4). Consequently, the stated properties of $N^{*}$ hold for all such closed loops. The following result is easily obtained. Assume that the closed loop input $w:[0, \infty) \rightarrow R^{p}$ is a given integrable function. If 
the initial condition data satisfy $x_{1} \in N^{*}$, then there exists a unique solution (at least locally defined) of the initial value problem corresponding to equations (3.2)-(3.3) which satisfies $x(t) \in$ $N^{*}$ for each $t$ for which the solution is defined. We subsequently use the notation

$\left(x\left(t, x_{1}, w\right), v\left(t, x_{1}, w\right)\right)$

to denote the solution of equations (3.2)-(3.3) at time $t \geqslant 0$ corresponding to the initial data $x_{1}$ and the closed loop input function $w:[0, \infty) \rightarrow R^{p}$.

A particularly important class of solutions are the equilibrium solutions. A solution of equations (3.2)-(3.3) is an equilibrium solution if it is a constant solution. Suppose that $w(t)=0, t \geqslant 0$ and that $\gamma_{i}(x), i=1, \ldots, p$, are smooth functions defined on $M$. Equations (3.2)-(3.3) have equilibria solutions defined by

$$
\begin{aligned}
& \left\{(x, v) \in N^{*} \times R^{m} \mid\right. \\
& f(x)+\sum_{i=1}^{p} h_{i}(x) \gamma_{i}(x)+\sum_{i=1}^{m} g_{i}(x) v_{i}=0 \\
& \left.k_{i}(x)=0, i=1, \ldots, m\right\}
\end{aligned}
$$

These equilibria solutions for equations (3.2)-(3.3) define a subset of $N^{*}$.

$$
\begin{aligned}
& N_{\mathrm{e}}=\left\{x \in N^{*} \mid f(x)+\sum_{i=1}^{p} h_{i}(x) \gamma_{i}(x)\right. \\
& \quad+\sum_{i=1}^{m} g_{i}(x) v_{i}=0, \\
& \left.\quad \text { for some } v \in R^{m}, k_{i}(x)=0, i=1, \ldots, m\right\},
\end{aligned}
$$

so that if $x_{1} \in N_{\mathrm{e}}$ then $x\left(t, x_{1}, 0\right)=x_{1}$ for all $t \geqslant 0$.

We now formulate a feedback stabilization problem for nonlinear constrained systems defined by differential-algebraic equations (2.4)(2.5); a suitable stability definition is first introduced for the closed loop differential-algebraic equations (3.2)-(3.3).

Definition 3.1. Assume that $w(t)=0, t \geqslant 0$, in the specification for the closed loop constrained system defined by equations (3.2)-(3.4). Let $x_{0} \in N_{\mathrm{e}}$.
Then $x_{0}$ is locally stable if for any neighborhood $U$ of $x_{0}$ there is a neighborhood $V$ of $x_{0}$ with $U \supset V$ such that if $x_{1} \in V \cap N^{*}$ then the solution of equations (3.2)-(3.4) satisfies

$x\left(t, x_{1}, 0\right) \in U \cap N^{*}$ for all $t \geqslant 0$.

If, in addition,

$x\left(t, x_{1}, 0\right) \rightarrow x_{0} \quad$ as $t \rightarrow \infty$

then we say that $x_{0}$ is locally asymptotically stable.

It is clear that if $x_{0}$ is a locally asymptotically stable equilibrium solution then it must be an isolated equilibrium on $N^{*}$. The following stabilizability concept is introduced.

Definition 3.2. The nonlinear differential-algebraic equations (2.4)-(2.6) are said to be locally stabilizable to $x_{0} \in N_{\mathrm{e}}$ if there exists a feedback function of the form given in (3.1) such that $x_{0}$ is a locally asymptotically stable equilibrium solution of the closed loop equations (3.2)-(3.4).

If there exists such a feedback function of the form (3.1) which is smooth on $N^{*}$ then we say that the constrained system defined by equations (2.4)-(2.6) is smoothly stabilizable to $x_{0}$. Note that Definition 3.2 requires that there exists a feedback function of the form (3.1) for which $x_{0}$ is at least an isolated equilibrium of the closed loop. The constrained problems considered in [2] represent a class for which no smooth stabilizing feedback exists but nonsmooth stabilizing feedback may exist. It is possible to define the notions of stability of a set of equilibria and stabilizability to a set of equilibria as in [2], but such generalizations are not considered here.

\section{A state realization approach to the local stabili- zation of systems described by nonlinear differen- tial-algebraic equations}

One approach to an analysis of the stabilizability problem is to introduce a coordinate transformation $(x, v) \rightarrow(\bar{x}, v)$ so that the constraints have a trivial specification; then the constraints are enforced so that a set of decomposed differential and algebraic equations for the constrained system 
are obtained: the reduced differential equations characterize the solution $\bar{x}\left(t, x_{1}, u\right)$ on the constraint manifold independently of $v$ and the algebraic equations characterize the solution $v\left(t, x_{1}, u\right)$ in terms of the solution $\bar{x}\left(t, x_{1}, u\right)$. Define

$$
\begin{aligned}
& \bar{x}_{1}=k_{1}(x), \\
& \bar{x}_{2}=L_{f} k_{1}(x), \\
& \quad \vdots \\
& \bar{x}_{r_{1}}=L_{f}^{r_{1}-1} k_{1}(x), \\
& \bar{x}_{r_{1}+1}=k_{2}(x), \\
& \quad \vdots \\
& \bar{x}_{r_{1}+\cdots+r_{m}}=L_{f}^{r_{m}-1} k_{m}(x) .
\end{aligned}
$$

It is possible to introduce functions

$\phi_{i}(x), \quad i=r_{1}+\cdots+r_{m}+1, \ldots, n$,

so that if

$\bar{x}_{i}=\phi_{i}(x), \quad i=r_{1}+\cdots+r_{m}+1, \ldots, n$,

then the mapping $x \rightarrow \bar{x}=\Phi(x)$ is a local diffeomorphism on $M \cap N\left(x_{0}\right)$ and $\bar{x}_{1}, \ldots, \bar{x}_{n}$ define local coordinates on $M \cap N\left(x_{0}\right)$. In terms of the transformed variables, the differential equations (2.1)-(2.3) are in the normal form

$$
\begin{aligned}
& \dot{\bar{x}}^{=} \bar{x}_{2}, \\
& \quad \vdots \\
& \dot{\bar{x}}_{r_{1}-1}=\bar{x}_{r_{1}}, \\
& \dot{\bar{x}}_{r_{1}}=L_{f}^{r_{1}} k_{1}(x)+\sum_{j=1}^{m}\left(L_{g_{j}} L_{f}^{r_{1}-1} k_{1}(x)\right) v_{j} \\
& \quad+\sum_{j=1}^{p}\left(L_{h_{j}} L_{f}^{r_{1}-1} k_{1}(x)\right) u_{j}, \\
& \dot{\bar{x}}_{r_{1}+1}=\bar{x}_{r_{1}+2}, \\
& \quad \vdots \quad \dot{\bar{x}}_{r_{1}+\cdots}+r_{m}=L_{f}^{r_{m}} k_{m}(x)+\sum_{j=1}^{m}\left(L_{g_{j}} L_{f}^{r_{m}-1} k_{m}(x)\right) v_{j} \\
& \quad+\sum_{j=1}^{p}\left(L_{h_{j}} L_{f}^{r_{m}-1} k_{m}(x)\right) u_{j},
\end{aligned}
$$

$$
\begin{aligned}
& \dot{\bar{x}}_{i}= L_{f} \phi_{i}(x)+\sum_{j=1}^{m}\left(L_{g_{j}} \phi_{i}(x)\right) v_{j} \\
&+\sum_{j=1}^{p}\left(L_{h_{j}} \phi_{i}(x)\right) u_{j}, \\
& i= r_{1}+\cdots+r_{m}+1, \ldots, n, \\
& y_{i}=\bar{x}_{r_{i-1}+1}, \quad i=1, \ldots, m, \\
& z_{i}=l_{i}(\bar{x}), \quad i=1, \ldots, p,
\end{aligned}
$$

where $r_{0}=0$. An equivalent representation of the constrained system defined by equations (2.4)(2.6) is obtained by imposing the constraints $y_{i}=0$, $i=1, \ldots, m$. These constraints are satisfied if

$\bar{x}_{i}(0)=0, \quad i=1, \ldots, r_{1}+\cdots+r_{m}$,

and if

$v_{i}=\alpha_{i}(x)+\sum_{j=1}^{p} \beta_{i j}(x) u_{j}, \quad i=1, \ldots, m$,

where

$$
\begin{aligned}
& {\left[\begin{array}{c}
\alpha_{1}(x) \\
\vdots \\
\alpha_{m}(x)
\end{array}\right]=-A^{-1}(x)\left[\begin{array}{c}
L_{f}^{\prime} k_{1}(x) \\
\vdots \\
L_{f}^{r} k_{m}(x)
\end{array}\right],} \\
& {\left[\begin{array}{ccc}
\beta_{11}(x) & \ldots & \beta_{1 p}(x) \\
\vdots & & \vdots \\
\beta_{m 1}(x) & \ldots & \beta_{m p}(x)
\end{array}\right]} \\
& =-A^{-1}(x)\left[\begin{array}{ccc}
L_{h_{1}} L_{f}^{r_{1}-1} k_{1}(x) & \ldots & L_{h_{p}} L_{f}^{r_{1}-1} k_{1}(x) \\
\vdots & & \vdots \\
L_{h_{1}} L_{f}^{r_{m}-1} k_{m}(x) & \ldots & L_{h_{p}} L_{f}^{r_{m}-1} k_{m}(x)
\end{array}\right] .
\end{aligned}
$$

It follows that

$\bar{x}_{i}(t)=0, \quad t \geqslant 0, \quad i=1, \ldots, r_{1}+\cdots+r_{m}$.

Thus $\bar{x}_{r_{1}+\ldots+r_{m}+1}, \ldots, \bar{x}_{n}$ define local coordinates on $N^{*}$ and the nonlinear constrained system is described in these local coordinates by the ordinary differential equations

$$
\begin{aligned}
\dot{\bar{x}}_{i}=\bar{f}_{i}(\dot{\bar{x}})+\sum_{j=1}^{p} \bar{h}_{i j}(\bar{x}) u_{i}, \\
\quad i=r_{1}+\cdots+r_{m}+1, \ldots, n, \\
z_{i}=\bar{l}_{i}(\bar{x}), \quad i=l, \ldots, p,
\end{aligned}
$$


where

$$
\begin{aligned}
\tilde{f}_{i}(\bar{x})= & {\left[L_{f} \phi_{i}(x)\right.} \\
& \left.+\sum_{k=1}^{m}\left(L_{g_{k}} \phi_{i}(x)\right) \alpha_{k}(x)\right]_{x=\Phi^{-1}(\bar{x})}, \\
\bar{h}_{i j}(\bar{x})= & {\left[L_{h_{j}} \phi_{i}(x)\right.} \\
& \left.+\sum_{k=1}^{m}\left(L_{g_{k}} \phi_{i}(x)\right) \beta_{k_{j}}(x)\right]_{x=\Phi^{-1}(\bar{x})}, \\
\bar{l}_{i}(\bar{x})= & l_{i}\left(\Phi^{-1}(\bar{x})\right) .
\end{aligned}
$$

The right hand sides of equations (4.1) and (4.2) can be expressed solely in terms of the transformed variables $\bar{x}_{r_{1}+\ldots+r_{m}+1}, \ldots, \bar{x}_{n}$ and $u_{1}, \ldots, u_{p}$. Consequently, equations (4.1)-(4.2) provide an $n-\left(r_{1}+\cdots+r_{m}\right)$ dimensional local nonlinear state realization for the nonlinear constrained system defined by the differential-algebraic equations (2.4)-(2.6).

One approach to the local stabilization of the nonlinear differential-algebraic equations (2.4)(2.5) depends on the classical linearization of the state realization given by equations (4.1)-(4.2). Suppose that $\left(x_{0}, v_{0}\right) \in N^{*} \times R^{m}$ is an equilibrium solution of equations (2.4)-(2.5) corresponding to $u=0$. Thus there is a corresponding vector $\overline{\mathrm{x}}_{0}=\left(0, \ldots, 0, \zeta_{0}\right)$ which is an equilibrium solution of equations (4.1)-(4.2) with $u=0$. Consider the linear differential equations, associated with the nonlinear differential equations (4.1)(4.2),

$\dot{\xi}=\bar{A} \xi+\sum_{j=1}^{p} \bar{c}_{j} \mu_{j}$

where

$$
\begin{aligned}
\vec{A} & =\left[\frac{\partial \bar{f}_{i}(\bar{x})}{\partial \bar{x}_{j}}, i, j=r_{1}+\cdots+r_{m}+1, \ldots, n\right]_{\bar{x}=\bar{x}_{0}}, \\
\bar{c}_{j} & =\left[\begin{array}{c}
\bar{h}_{r_{1}+\cdots+r_{m}+1, j}\left(\bar{x}_{0}\right) \\
\vdots \\
\bar{h}_{n, j}\left(\bar{x}_{0}\right)
\end{array}\right] .
\end{aligned}
$$

The following result is based on classical arguments.
Theorem 4.1. If the linear differential-algebraic equations (4.3) are stabilizable then the equilibrium solution $\left(x_{0}, v_{0}\right)$ of the nonlinear differential-algebraic equations (2.4)-(2.5) is locally stabilizable.

Corollary 4.2. If each eigenvalue of the matrix $\bar{A}$, with real part that is nonnegative, is controllable (according to equations (4.3)) then the equilibrium solution $\left(x_{0}, v_{0}\right)$ of the nonlinear differential-algebraic equations (2.4)-(2.5) is locally stabilizable.

General conditions for the existence of a local stabilizing controller for the constrained system defined by the differential-algebraic equations (2.4)-(2.5), which hold even in the critical cases not covered by the assumptions in Theorem 4.1, can be developed using the state realization given by equations (4.1)-(4.2). In particular, we mentioned that if equations (4.1)-(4.2) define a minimum-phase nonlinear system which satisfies a certain invertibility condition as defined in [4] then it, and hence also the constrained system defined by the differential-algebraic equations (2.4)-(2.5), can be locally stabilized.

Theorem 4.1 and Corollary 4.2 are important results, but their practical utility depends on construction of a nonlinear diffeomorphism required to obtain a state realization.

\section{A classical linearization approach to the local stabilization of systems described by nonlinear dif- ferential-algebraic equations}

We now extend the classical approach to local stabilization based on the use of linearized equations to the nonlinear differential-algebraic equations (2.4)-(2.6). We develop a set of linear differential-algebraic equations that represent a first order approximation to the nonlinear differentialalgebraic equations (2.4)-(2.6) at an equilibrium, and we demonstrate that, under certain assumptions, if the linearized equations can be stabilized then the nonlinear constrained system can be locally stabilized to the equilibrium solution. Such a plan is similar to recent results in [16] for a more general class of behavioral differential equations that are not in state variable form. 
Suppose that $\left(x_{0}, v_{0}\right) \in N^{*} \times R^{m}$ is an equilibrium solution of the differential-algebraic equations (2.4) $-(2.6)$ corresponding to $u=0$. That is,

$f\left(x_{0}\right)+\sum_{i=1}^{m} g_{i}\left(x_{0}\right) v_{0 i}=0$,

$0=k_{i}\left(x_{0}\right), \quad i=1, \ldots, m$.

Introduce the linear constrained system defined by the linear differential-algebraic equations

$\dot{\eta}=A \eta+\sum_{i=1}^{m} b_{i} \zeta_{i}+\sum_{i=1}^{p} c_{i} \omega_{i}$

$\theta_{i}=d_{i} \eta=0, \quad i=1, \ldots, m$,

$\varphi_{i}=f_{i} \eta, \quad i=1, \ldots, p$,

where

$A=\left[\frac{\partial}{\partial x}\left[f(x)+\sum_{i=1}^{m} g_{i}(x) v_{i}\right]\right]_{x=x_{0}, v=v_{0}}$,

$b_{i}=g_{i}\left(x_{0}\right), \quad i=1, \ldots, m$,

$c_{i}=h_{i}\left(x_{0}\right), \quad i=1, \ldots, p$,

$d_{i}=\left[\frac{\partial}{\partial x} k_{i}(x)\right]_{x=x_{0}}, \quad i=1, \ldots, m$,

$f_{i}=\left[\frac{\partial}{\partial x} l_{i}(x)\right]_{x=x_{0}}, \quad i=1, \ldots, p$.

If Assumptions (A1)-(A3) hold at $x_{0}$ it can be shown, using the normal form equations, that

$$
\begin{aligned}
& d_{i} A^{k} b_{j}=0, \quad k=0, \ldots, r_{i}-2, i=1, \ldots, m, \\
& j=1, \ldots, m, \\
& \operatorname{rank}\left[d_{i} A^{r_{i}-1} b_{j}\right]=m, \\
& d_{i} A^{k} c_{j}=0, \quad k=0, \ldots, r_{i}-2, i=1, \ldots, m, \\
& j=1, \ldots, p .
\end{aligned}
$$

Consequently, the following result is a corollary of Theorem 2.1 for the linear differential-algebraic equation (5.3)-(5.5).

\section{Corollary 5.1. Define}

$$
\begin{array}{r}
S^{*}=\left\{\eta \in R^{n} \mid d_{i} A^{k} \eta=0, k=0, \ldots, r_{i}-1,\right. \\
i=1, \ldots, m\} .
\end{array}
$$

Then $S^{*}$ is an $n-\left(r_{1}+\cdots+r_{m}\right)$ dimensional subspace of $R^{n}$ which is the maximal controlled invariant subspace contained in $\operatorname{ker}\left\{d_{1}, \ldots, d_{m}\right\}$.
Assume that the input function $\omega:[0, \infty) \rightarrow R^{p}$ is a given integrable function. If the initial condition data satisfy $\eta_{1} \in S^{*}$, then there exists a unique solution of the initial value problem defined by the linear differential-algebraic equations (5.3)-(5.5) and the solution satisfies $\eta(t) \in S^{*}$.

The main result of this section is stated in the following theorem.

Theorem 5.2. If the linear differential-algebraic equations (5.3)-(5.4) are stabilizable (as defined in Definition 3.2) then the equilibrium solution $\left(x_{0}, v_{0}\right)$ of the nonlinear differential-algebraic equations (2.4)-(2.5) is locally stabilizable.

Proof. Note that the tangent plane of $N^{*}$ at $x_{0}$ is a translation of the subspace $S^{*}$. The proof follows according to [5] and the facts that equations (2.4)-(2.5) define a smooth vector field on the manifold $N^{*}$ and that the linearized vector field on the tangent plane of $N^{*}$ at $x_{0}$ is exactly the linear vector field defined by equations (5.3)-(5.4) on $S^{*}$.

The design of a linear stabilizing controller for the linear differential-algebraic equations (5.3)(5.4) is nonstandard. One effective approach is to construct the (linear in this case) diffeomorphism developed in Section 4 to obtain a linear state realization for the linearized differential-algebraic equations (5.3)-(5.4). The linear state realization thereby obtained is precisely equation (4.3). This approach has been developed in [7] into a direct computational procedure, avoiding construction of any nonlinear diffeomorphism, to solve the local feedback stabilization problem. The main part of the computations to obtain the linear state realization (4.3) involves a singular value decomposition. Application of this approach to local stabilization of a class of constrained robot problems is described in [8].

\section{References}

[1] A.M. Bloch, Stabilizability of nonholonomic control systems, Proceedings of MTNS-89 (Birkhäuser, Basel-Boston, 1989).

[2] A. Bloch and N.H. McClamroch, Control of mechanical systems with classical nonholonomic constraints, Proceedings of IEEE Conference on Decision and Control, Tampa, FL (1989) 201-205. 
[3] K.E. Brenan, S.L. Campbell and L.R. Petzold, Numerical Solution of Initial-Value Problems in Differential-Algebraic Equations (Springer-Verlag, New York, 1989).

[4] C.I. Byrnes and A. Isidori, Local stabilization of minimum-phase nonlinear systems, Systems Control Lett. 11 (1988) 9-17.

[5] D.J. Chillingworth, Differential Topology with a View to Applications (Pitman, London, 1977).

[6] A. Isidori, Nonlinear Control Systems, Second Edition (Springer-Verlag, Berlin-New York, 1989).

[7] H. Krishnan and N.H. McClamroch, On control systems described by a class of linear differential-algebraic equations: state realizations and linear quadratic optimal control, Proceedings of American Control Conference, San Diego, CA (1990).

[8] H. Krishnan and N.H. McClamroch, A new approach to regulation of force and position in constrained robot systems, Proceedings of 1990 Conference on Robotics and Automation, Cincinnati, OH (1990).

[9] R. Marino, W. Respondek and A.J. van der Schaft, Almost disturbance decoupling for single-input-output nonlinear systems, IEEE Trans. Automat. Control 34 (1989) 1013-1017.

[10] N.H. McClamroch, On control systems described by a class of nonlinear differential-algebraic equations: state realizations and local control, Proceedings of American Control Conference, San Diego, CA (1990).

[11] N.H. McClamroch and D. Wang. Feedback stabilization and tracking of constrained robots, IEEE Trans. Automat. Control 33 (1988) 419-426.

[12] J.K. Mills and A.A. Goldenberg, Force and position control of manipulators during constrained tasks, IEEE Trans. Robotics and Automat 5 (1989) 30-46.

[13] C.C. Pantelides, The consistent initialization of differential-algebraic systems, SIAM J. Sci. Stat. Comput. 9(1988) 213-221.

[14] W.C. Rheinboldt, Differential-algebraic systems as differential equations on manifolds. Math. Comput. 43 (1984) 473-482.

[15] A.J. van der Schaft, On clamped dynamics of nonlinear systems, in: C.I. Byrnes, C.F. Martin and R.E. Saeks, Eds., Analysis and Control of Nonlinear Systems (NorthHolland, Amsterdam, 1988) 499-506.

[16] J.C. Willems and F. Lamnabhi-Lagarrigue, A note on the linearization around an equilibrium, Proceedings of IEEE Conference on Decision and Control, Tampa, FL (1989) 2259-2261. 